\title{
The Effect of Inhaled Cannabis on Intraocular Pressure in Healthy Adult Subjects
}

\author{
Sameh Mosaed, ${ }^{1,2}$ John HK Liu, ${ }^{3}$ Donald S Minckler, ${ }^{2}$ Robert L Fitzgerald, ${ }^{4}$ David Grelotti, ${ }^{5}$ Emily Sones, ${ }^{5}$ Catherine R \\ Sheils, ${ }^{1}$ Robert N Weinreb ${ }^{3}$ and Thomas D Marcotte ${ }^{5}$ \\ 1. Gavin Herbert Eye Institute, University of California, Irvine, CA, USA; 2. Irvine School of Medicine, University of California, Irvine, CA, USA; \\ 3. Viterbi Family Department of Ophthalmology, University of California, San Diego, CA, USA; 4. Department of Pathology, University of \\ California, San Diego, CA, USA; 5. Department of Psychiatry, University of California, San Diego, CA, USA
}

DOI: https://doi.org/10.17925/OPHT.2021.15.1.33

$\mathrm{P}$ urpose: To study the effect of inhaled cannabis on intraocular pressure (IOP) in healthy adult subjects. Design: Prospective, doubleblind, randomized, placebo-controlled trial. Methods: Study participants were randomized to receive a single dose of inhaled cannabis or placebo in an 11:3 ratio. Subjects self-administered inhaled cannabis or placebo cigarette in negative-pressure rooms. Measurements of IOP, systemic blood pressure and plasma tetrahydrocannabinol levels were taken at baseline and every 40 minutes for 2 hours, and afterwards, every hour for 3 hours. A linear mixed-effects models and Wald tests were performed on the IOP readings and systemic blood pressure measurements to compare the treatment group with the placebo group, and with baseline readings, using $p$-value significance of alpha $\leq 0.05$. Results: A total of 14 participants were included. Average baseline IOP for the treatment group and placebo group was $17.5 \mathrm{mmHg}$ and $18.4 \mathrm{mmHg}$, respectively, and did not significantly differ between the groups. IOP was significantly lowered from baseline in the treatment group at all time points post-treatment, up to the 4 -hour time point $(p<0.01)$. The placebo group had extensive IOP fluctuations and had a significant IOP reduction only at the 80 -minute, 3-hour and 5 -hour time points $(p<0.05)$. Peak IOP lowering occurred at the 80 -minute time point with $15.7 \%$ reduction in IOP in the treatment group. There were no significant changes in systemic blood pressure during the duration of the study. Conclusions: Cannabis lowers IOP significantly from baseline in adult healthy subjects. This effect is detected from 40 minutes post-treatment and lasts up to 4 hours. Systemic blood pressure was not significantly affected.

\section{Keywords}

Cannabis and glaucoma, intraocular pressure, tetrahydrocannabinol

Disclosures: Sameh Mosaed, John HK Liu, Donald S Minckler, Robert L Fitzgerald, David Grelotti, Emily Sones, Catherine R Sheils, Robert N Weinreb, and Thomas D Marcotte have no financial or non-financial relationships or activities to declare in relation to this article.

Acknowledgments: This study was conducted using departmental funds from the University of California, Irvine, Gavin Herbert Eye Institute. Statistical review was provided by Alex Noble, Touch Independent Medical Education.

Review Process: Double-blind peer review.

Compliance with Ethics: All procedures were followed in accordance with the responsible committee on human experimentation and with the Helsinki Declaration of 1975 and subsequent revisions, and informed consent was received from the patient involved in this case study. Authorship: The named authors meet the Internationa Committee of Medical Journal Editors (ICMJE) criteria for authorship of this manuscript, take responsibility for the integrity of the work as a whole, and have given final approval for the version to be published.

Access: This article is freely accessible at touchOPHTHALMOLOGY.com (c) Touch Medical Media 2020

Received: 10 August 2020

Accepted: 1 December 2020

Published Online: 24 May 2021

Citation: touchREVIEWS in Ophthalmology. 2021;

15(1):33-7

Corresponding Author: Sameh Mosaed, Gavin Herbert Eye Institute, University of California, 850 Health Sciences Road, Irvine, CA 92697-4375, USA. E: smosaed@hs.uci.edu

Support: This study was conducted using departmental funds from the University of

California, Irvine Gavin Herbert Eye Institute.
The effect of cannabis on intraocular pressure (IOP) has long been a subject of speculation. Indeed, the first legal use of cannabis for any medical purpose in the USA was granted for the treatment of a patient with glaucoma in 1976 (United States v. Robert Randall, D.C. Super. Ct., 1974). In the half century since then, only a few small studies have attempted to characterize the effects of cannabis on IOP. Cannabis is a genus of flowering plants in the Cannabaceae family, which consists of three primary species: Cannabis sativa, Cannabis indica and Cannabis ruderalis. Cannabis contains more than 480 different compounds, of which more than 100 have been identified as phytocannabinoids, including cannabidiol (CBD), and tetrahydrocannabinol $\left(\Delta^{9}-\mathrm{THC}\right){ }^{1,2}$ Cannabis is classified as a schedule $1 \mathrm{drug}$ by the USA Drug Enforcement Administration (along with heroin and LSD [lysergic acid diethylamide]). Accordingly, there are many barriers to research involving cannabis and its derivatives on human subjects. ${ }^{3}$

Early, non-randomized studies in the 1970s and early 1980s showed that inhaled cannabis lowered IOP by about $25 \%$, and this effect was shown to last between 2 and 4 hours. ${ }^{4} A$ study by Cooler and Greg in 1977, using intravenously injected THC in 10 human subjects, found that IOP was lowered by an average of $37 \% .{ }^{5}$ Topically applied THC has been shown to reduce IOP by $28 \%$ in a recent animal study, whereas topical CBD was shown to elevate IOP. ${ }^{6}$ Another small study suggested that sublingually administered THC lowered IOP for up to 4 hours in a group of six human subjects.? Although these and other published studies have confirmed that THC lowers IOP, this has not translated to the adoption or use of THC in clinical practice. There are many reasons for this. Firstly, cannabis is a psychoactive drug and systemic administration poses limitations for most patients. In addition, other side effects, such as tachycardia and elevated systolic blood pressure, have been reported. ${ }^{8-10}$ Topically delivered formulations have historically poor penetration through the cornea, and resultant lack of efficacy.11,12 Hence, our understanding of the impact of cannabis on IOP in humans has been limited to these scant reports in the literature over the decades. This is the first prospective, randomized, double-blind, placebo-controlled study evaluating the effect of inhaled cannabis on IOP in healthy human subjects.

\section{Methods}

\section{Regulatory process}

This study was approved by the by the University of California, San Diego Human Research Protections, and was in accordance with the Helsinki Declaration, and informed consent was 
obtained from all participants. The parent study was conducted under IND 131268, and approved by the Research Advisory Panel of California (ClinicalTrials.gov Identifier: NCT02849587). This study was conducted at the University of California, Center for Medicinal Cannabis Research (CMCR). Two rooms were specifically outfitted with a negative-pressure system to enable cannabis to be vented to the atmosphere without contaminating the workspace of others. Cannabis was harvested at the University of Mississippi under the supervision of the National Institute on Drug Abuse (NIDA). Bulk placebo was made from whole plant with extraction of cannabinoids, in particular THC, and has the natural smell and appearance of the active cannabis.

\section{Participant selection}

Participants were a consecutive subset of individuals enrolled in a larger study (recruited from the community) examining the impact of acute cannabis ingestion on driving performance. Patients had to be older than 21 years, a regular cannabis smoker ( $\geq 4$ times in the past month), and willing to complete the IOP tonometer evaluations. Exclusion criteria included a known history of glaucoma or other eye disorder other than refractive error; the inability to not use contact lenses on the day of visits; history of traumatic brain injury; an unwillingness to abstain from cannabis for 2 days prior to screening visit (so driving simulation will not be impaired) and 2 days prior to experimental visit (2-3 half-lives of THC); a positive pregnancy test; and a positive result on toxicity screening for cocaine, amphetamines, opiates and phencyclidine (PCP). However, a positive result for a prescribed or recommended drug (cannabis) was not exclusionary. Individuals with current substance use disorders, as assessed using the Drug Abuse Screening Test (DAST) and Alcohol Use Disorders Identification Test (AUDIT), were excluded. Subjects were also excluded for being unwilling to be transported by cab or have a responsible adult drive them home after experimental session or inability to complete study procedures (i.e. unwillingness to be transported home by taxi or friend). Eligible participants were scheduled for a baseline session and one 8-hour experimental session at the CMCR.

\section{Study design}

A subgroup of participants, from the parent study of 300 participants, was screened and enrolled for this IOP sub analysis. At the beginning of the screening/baseline visit and the experimental visit, subjects underwent a urine drug screen and breathalyzer for alcohol and drugs. In addition, an oral fluid sample was run for the presence of $\triangle 9$-THC using a testing device (Dräger DrugTest ${ }^{\circledR} 5000$, Dräger, Lübeck, Germany) which identifies THC levels at above versus below $5 \mathrm{ng}$. A positive reading on the Draeger was considered indicative of use within the past day. Any participants with a positive reading were excluded (none occurred within the subsample for this study).

Participants were divided into two groups, receiving either $5.9 \%$ or $13.4 \%$ $\Delta^{9}$-THC, or placebo cannabis cigarettes at their visits. Participants were given $700 \mathrm{mg}$ cigarettes with either $0 \%$ (placebo), or $5.9 \%$ or $13.4 \% \Delta^{9}$ THC (treatment drug) at the beginning of the day and were instructed to "smoke the cigarette the way you do at home to get high" (i.e. there was no requirement that they finish the entire cigarette). Though not mandatory to incinerate the cigarette to the proximal tip, a minimum of four puffs was required for a participant to remain in the study. They were allowed 10 minutes to smoke. The allocation schedule was kept in the pharmacy and concealed from other study personnel. Patients and assessors were blinded to group assignments. IOP measurements and bodily fluid draws (e.g. blood, oral fluid saliva, breath) were measured before the cannabis smoking and over the subsequent 6 hours after cannabis smoking.
Vital signs were checked to monitor the subjects' health status as well as to quantify the general effects of the cannabis. Blood pressure and pulse were assessed pre-smoking, and at approximately every 30 minutes for 2 hours post smoking session, then up to every hour for the additional 3 hours. At any sign of an adverse reaction (e.g. a change in blood pressure or pulse rate, or development of psychological distress), an investigator was called. Subjects remained in the laboratory under direct observation for 7 hours after the smoking inhalations were completed. At that time, a final vital sign and self-report status check was made, and upon satisfactory readings, each subject was released and driven back to their domicile by taxicab or prearranged transportation. The return transport procedure was observed directly by staff to ensure compliance.

IOP measurements were taken using the non-contact Ocular Response Analyzer ${ }^{\circledR}$ (Reichert Technologies, Depew, NY, USA). This device is FDAapproved and was used in accordance with the FDA label. Three IOP readings were taken for each eye prior to smoking, with the average value calculated, and then at approximately 40 minutes, 80 minutes, 120 minutes and every hour for the additional 3 hours after smoking. IOP readings were obtained by trained research assistants. If the participant had high IOP ( $\geq 21 \mathrm{mmHg}$ ) prior to smoking, we recommended follow-up with an ophthalmologist.

\section{Analysis}

For the IOP analysis, a paired t-test was used to compare mean IOP at baseline between the treatment and placebo groups. A linear mixed effect model was used to evaluate change in intraocular pressure over time in the treatment and placebo groups. Linear mixed effect models are particularly useful in settings where repeated measurements are made on the same statistical units. A Wald test was used at each point of follow-up to determine whether the average IOP had changed from baseline. The $p$-value significance level was set at alpha $\leq 0.05$. Three IOP readings were taken in each eye from each subject, at each time period studied, and the average of the three readings from each eye was calculated. For the analysis of blood pressure, a linear mixed effect model was again used, and wald tests repeated at each point of followup, with the same p-value significance level. The systolic and diastolic blood pressure recordings were obtained from each subject at baseline and each time point and included in the analysis.

\section{Results}

The age range of study participants was 21-55 years. Data from 28 eyes (14 patients) were analyzed. The data were divided into two groups: treatment ( $n=22$ eyes) and placebo ( $n=6$ eyes). There were no missing values or outliers in the data, and the IOP was normally distributed. Because the participants were allowed to smoke a discretionary amount to achieve psychotropic effects, the low-dose and high-dose group had similar plasma levels of THC and were therefore grouped together in one treatment group.

At baseline, there was no significant difference between the mean IOP in the treatment group $(17.5 \mathrm{mmHg}$ ) and the placebo group (18.4 $\mathrm{mmHg}$ ) using a paired t-test $(p=0.584)$. In the treatment group, mean intraocular pressure decreased to $14.9 \mathrm{mmHg}$ at 40 minutes, $14.7 \mathrm{mmHg}$ at 80 minutes, $15.0 \mathrm{mmHg}$ at 2 hours, $15.5 \mathrm{mmHg}$ at 3 hours, $15.3 \mathrm{mmHg}$ at 4 hours, and $16.2 \mathrm{mmHg}$ at 5 hours (Figure 1). In the placebo group, mean intraocular pressure decreased to $18.1 \mathrm{mmHg}$ at 40 minutes, $15.8 \mathrm{mmHg}$ at 80 minutes, $17.1 \mathrm{mmHg}$ at 2 hours, $15.3 \mathrm{mmHg}$ at 3 hours, $15.9 \mathrm{mmHg}$ at 4 hours and $16.4 \mathrm{mmHg}$ at 5 hours (Figure 1). In the treatment group, the IOP reduction was statistically significant at all time points after treatment, up to and including 4 hours post-treatment, using the Wald Test on the linear mixed effects model ( $p<0.01$ at all time points) (Figure 1). 
Figure 1: Mean intraocular pressure over time across different groups

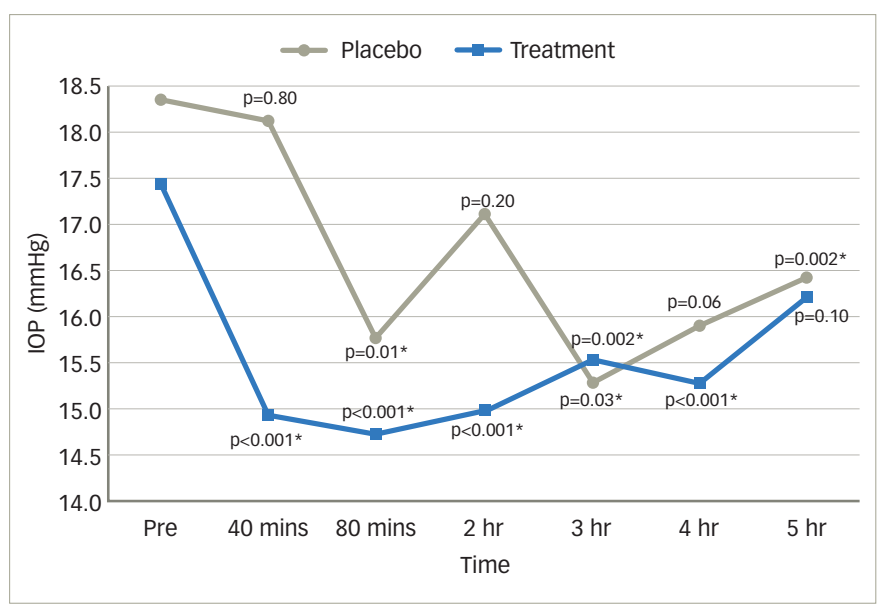

$P$-values represent difference at each time point from baseline. $1 O P=$ intraocular pressure.

Figure 2: Percentage intraocular pressure reduction over time

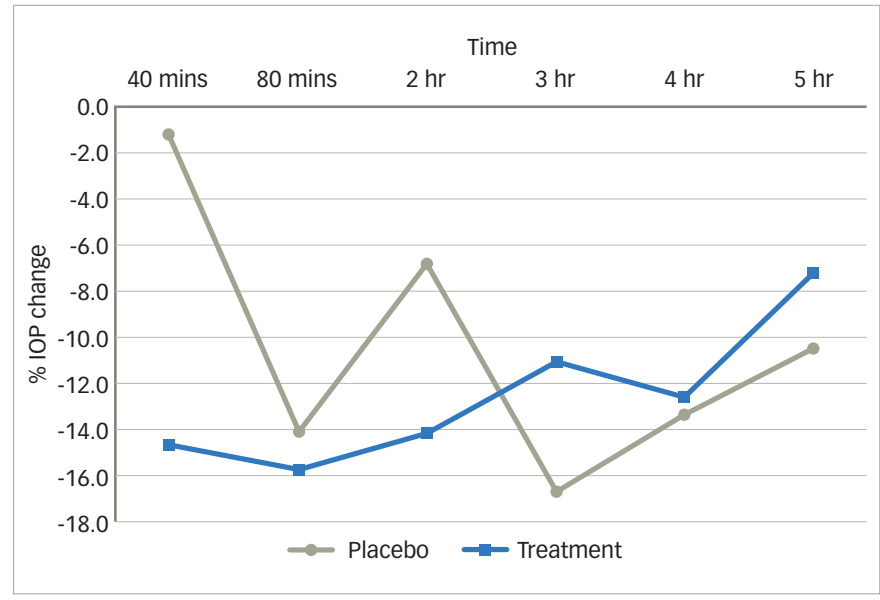

$1 O P=$ intraocular pressure.

There was also a significantly lowered IOP in the placebo group, but this was only seen at variable intervals: at the 80-minute, 3-hour and 5-hour time points $(p<0.05)$. In the treatment group, the percentage decrease in IOP from baseline was noted to be $14.6 \%$ at 40 minutes, $15.7 \%$ at 80 minutes and $14.1 \%$ at 2 hours (Figure 2). In the placebo group, the percentage decrease in IOP from baseline was noted to be $1.2 \%$ at 40 minutes, $14.1 \%$ at 80 minutes and $6.8 \%$ at 2 hours (Figure 2 ).

Blood pressure was measured in 15 patients. Linear mixed-effect models and Wald tests were used to analyze the data. In the placebo group, the change in systolic and diastolic blood pressure at 80 minutes was statistically significant compared to baseline $(p<0.05)$, but was not significant at any other time point measured (Figures 3 and 4). In the treatment group, the change in diastolic blood pressure at 2 hours was statistically significant compared to baseline $(p<0.05)$, but there was no significant effect on systolic or diastolic blood pressure at any other time point measured (Figures 3 and 4).

\section{Discussion}

The results of this study indicate that in healthy adult subjects, inhaled cannabis significantly lowers IOP from baseline, and that this effect is
Figure 3: Mean systolic blood pressure over time

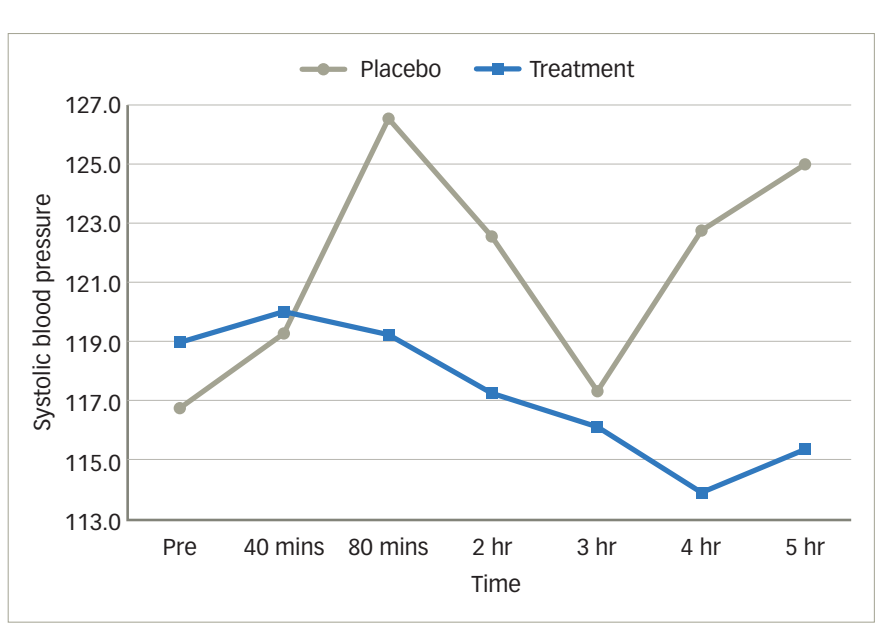

Figure 4: Mean diastolic blood pressure over time

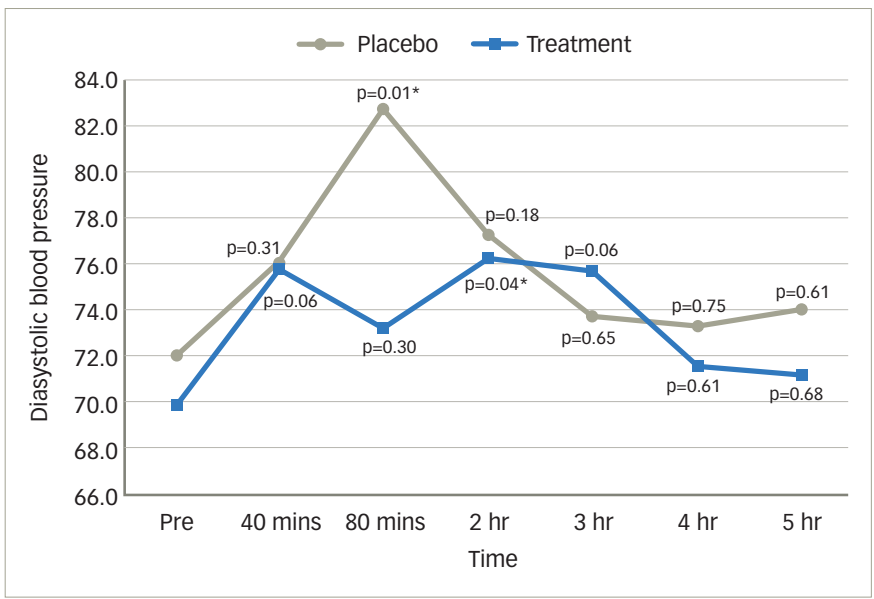

P-values represent difference at each time point from baseline.

detected within 40 minutes of inhalation. This IOP lowering of about 15\% slowly drifted back towards baseline, but the effects were still significant at 4 hours post-treatment dosing. Interestingly, the placebo group also saw a reduction in IOP after dosing; however, the IOP effect was less dramatic, and more labile. There are several reasons for this.

First, there was a very small sample of participants in the placebo group, leading to less robust powering of the placebo arm. Second, the baseline IOP was lower in the treatment group ( $17.5 \mathrm{mmHg}$ ) compared to baseline IOP in the placebo group (18.4 mmHg); starting at a lower IOP limits the potential for further IOP reduction and skews the data such that a significant difference is more difficult to establish. Third, there may also have been an effect of relaxation imparted to the placebo group, as the treatment was self-administered in a quiet room without interruptions. Indeed, a recent study by Dada et al. concluded that a 21-day practice of relaxation and mindful meditation can lower IOP by $25 \% \cdot{ }^{14}$ Additionally, the placebo effect may have played a role in lowering IOP; it has been shown that endocannabinoid system can elicit a strong placebo effect, which can be activated by the smell of cannabis and visual cues associated with smoking cannabis, such as the cigarette itself, the smoke or the lighter. ${ }^{13}$ It can be assumed that when smoking what the subject understands might contain cannabis, a certain degree of expectation and relaxation is imparted, activating the endocannabinoid system, in turn, eliciting the placebo effect on IOP. Other studies of glaucoma 
pharmacotherapies have also demonstrated an effect of placebo on IOP. ${ }^{15}$ A recent article by Sharpe et al. reviewed the placebo-group IOP data from 23 clinical trials and found that in 22 of the trials, there was a significant effect of placebo on IOP, sometimes as much as $2 \mathrm{mmHg}$, similar to our findings. This meta-analysis of phase I/II glaucoma clinical trials investigating the placebo effect on IOP, found that IOP reduction from baseline was less pronounced in the treatment group than in the placebo group in all 50 studies reviewed. The authors concluded that in early-phase clinical trials, the reduction from baseline better approximates the results of later regulatory and post-commercialization trials than the decrease from placebo..$^{15}$

The specific mechanisms by which cannabis lowers IOP are the subject of active investigation. It is known that there are cannabinoid receptors located throughout the eye, in particular in the ciliary muscle, ciliary epithelium, trabecular meshwork and Schlemm's canal. ${ }^{16}$ These receptors, part of the endocannabinoid system, include $\mathrm{CB} 1, \mathrm{CB} 2$, GPR18, GPR119 and GPR55. It has been shown, in mouse models, that CB1, GPR18 and GPR119 each lower IOP when activated by topical administration of $\Delta^{9}$-THC. ${ }^{5}$ This study also found that CBD blocks THC from lowering IOP, hence the active IOP-lowering component of cannabis is $\Delta^{9}-\mathrm{THC}$, and not CBD.

Studies have found that CB1 activation results in a series of varied changes, such as ciliary body contraction, widening of schlemm's canal and activation of matrix metalloproteinase, which enhances outflow of the trabecular meshwork. ${ }^{17,18}$ Moreover, cannabinoids not only act through the CB1 pathway, but also activate COX-2, which increases the presence of prostaglandin E2 and metalloproteinases, enhancing the outflow of aqueous humor and thereby reducing $I P^{19,20}$ It has also been suggested that some cannabinoids lower IOP through a prostaglandin-mediated mechanism, as well as through adrenergic receptors within the eye..$^{21-23}$ There is a possibility that cannabis may enhance ocular blood flow and perhaps perfusion to the optic nerve. This was suggested by a study investigating the effect of oral dronabinol in healthy volunteers, which showed decreased retinal arterio-venous passage time, as measured by fluorescein angiography. ${ }^{24}$ Besides any manipulation on ocular blood flow, the potential role of cannabis in neuroprotection has been suggested by many studies, although no clear evidence of neuroprotection in glaucoma has yet been established. ${ }^{25,26}$
Aside from psychotropic effects, another potential limitation to the use of systemically administered cannabis is the purported effect on systemic blood pressure. The cardiovascular effects of cannabis are not well understood as they appear to be variable and heterogenous. Cannabis consumption has been shown to cause an increase in systolic blood pressure, while potentiating orthostatic hypotension and tachycardia. ${ }^{27}$ The ultimate effect of cannabis on perfusion pressure to the optic nerve remains unknown at this time. Data from the treatment group in the current study showed no significant effect on systolic blood pressure at any time point, and only one statistically significant diastolic blood pressure reading at 2 hours post-dose, which is not clinically significant, nor statistically different from placebo. These data did not raise any new concerns with regards to short-term cardiovascular side effects in healthy individuals.

Given the wide range of systemic considerations, the routine use of inhaled or ingested cannabis for glaucoma treatment is not generally feasible. However, novel compounds with improved corneal penetration are being developed for topical administration, thereby mitigating systemic side-effects. ${ }^{28,29}$ In addition, non-psychotropic cannabinoids and other CB1 receptor targets are being investigated for potential treatments that avoid systemic effects. ${ }^{30,31}$

There are several limitations to this study. First, the number of subjects in the placebogroup was small and may have introduced error into the results from the natural variability in IOP during the diurnal period. Second, the method of obtaining the IOP data was through a non-contact tonometry method in order to facilitate the acquisition of IOP data in contact lens wearers, and to decrease the invasiveness of measurements for this pilot study. Non-contact tonometry may overestimate IOP when compared to gold-standard applanation tonometry. ${ }^{32}$ Ideally, future studies should use more consistently accurate methods of tonometry. In addition, this study only involved healthy adults, and does not characterize the IOP-lowering effects of cannabis in subjects with glaucoma or ocular hypertension, or in subjects with concomitant IOP-lowering medications.

In conclusion, the current study demonstrates a significant IOP reduction with inhaled cannabis in healthy adults that is sustained for up to 4 hours, with no significant effect on cardiovascular parameters. Defining the role of cannabis in glaucoma treatment requires further studies to better characterize these effects in different patient populations. •
1. Morales P, Hurst DP, Reggio PH. Molecular targets of the phytocannabinoids: a complex picture. Prog Chem Org Nat Prod. 2017;103:103-31

2. EISohly MA, Waseem G, Constituents of cannabis sativa. In: Pertwee R (ed), Handbook of Cannabis. Oxford: Oxford Scholarship Online, 2016;3-22.

3. National Academies of Sciences, Engineering, and Medicine; Health and Medicine Division; Board on Population Health and Public Health Practice; Committee on the Health Effects of Mariiuana: An Evidence Review and Research Agenda. Th of Marijuana: An Evidence Review and Research Agenda. The Health Effects of Cannabis and Cannabinoids: The Current State of Evidence and Recommendations for Research. Washington (DC): National Academies Press (US); 2017 Jan 12. 15 , Challenges and Barriers in Conducting Cannabis Research. Available at: www.ncbi.nlm.nih.gov/books/ NBK425757/ (accessed 12 December 2020.

4. Hepler RS, Frank IR. Marihuana smoking and intraocular pressure. JAMA. 1971;217:1392

5. Cooler P, Gregg JM. Effect of delta-9-tetrahydrocannabino on intraocular pressure in humans. South Med 1 . 1977;70:951-4.

6. Miller S, Daily L, Leishman E, et al. $\Delta 9$-Tetrahydrocannabino and cannabidiol differentially regulate intraocular pressure. Invest Ophthalmol Vis Sci. 2018;59:5904-11.

7. Tomida I, Azuara-Blanco A, House $\mathrm{H}$, et al. Effect of sublingual application of cannabinoids on intraocular pressure: a pilot study. I Glaucoma. 2006;15:349-53.

8. Alshaarawy O, Elbaz HA. Cannabis use and blood pressure levels: United States National Health and Nutrition Examination Survey, 2005-2012. J Hypertens. 2016;34: 1507-12.
9. Malinowska B, Toczek M, Pedzinska-Betiuk A, Schlicker E. Cannabinoids in arterial, pulmonary and portal hypertension - mechanisms of action and potential therapeutic significance. Br J Pharmacol. 2019;176:1395-411.

10. Singh A, Saluja S, Kumar A, et al. Cardiovascular complications of marijuana and related substances: a review. Cardiol Ther. 2018;7:45-59.

11. Green K, Roth M. Ocular effects of topical administration of delta 9-tetrahydrocannabinol in man. Arch Ophthalmol. 1982;100:265-7.

12. Jay WM, Green K. Multiple-drop study of topically applied $1 \%$ delta 9-tetrahydrocannabinol in human eyes. Arch Ophthalmol. 1983:101:591-3.

13. Campbell NK. Medical marijuana research. CMAJ. 2016;188:822.

14. Dada T, Mittal D, Mohanty K, et al. Mindfulness meditation reduces intraocular pressure, lowers stress biomarkers and modulates gene expression in glaucoma: a randomized controlled trial. J Glaucoma. 2018;27:1061-7.

15. Sharpe RA, Nelson LA, Stewart JA, Stewart WC. Intraocula pressure efficacy of glaucoma medications versus placebo in phase II compared to later phase trials. Br J Ophthalmol. 2013;97:121-5.

16. Straiker AJ, Maguire G, Mackie K, Lindsey J. Localization of cannabinoid CB1 receptors in the human anterior eye and retina. Invest Ophthalmol Vis Sci. 1999;40:2442-8.

17. Panahi $Y$, Manayi $A$, Nikan M, Vazirian $M$. The arguments for and against cannabinoids application in glaucomatous for and against cannabinoids application in glaucom
retinopathy. Biomed Pharmacother. 2017:86:620-7.

18. Rosch S, Ramer R, Brune K, Hinz B. R(+)-methanandamide and other cannabinoids induce the expression of cyclooxygenase-2 and matrix metalloproteinases in human nonpigmented ciliary epithelial cells. J Pharmacol Exp Ther 2006;316:1219-28.

19. Kozak KR, Rowlinson SW, Marnett $\sqcup$. Oxygenation of the endocannabinoid, 2-arachidonylglycerol, to glyceryl prostaglandins by cyclooxygenase-2. J Biol Chem . 2000;275:33744-9.

20. Rouzer CA, Marnett L. Endocannabinoid oxygenation by cyclooxygenases, lipoxygenases, and cytochromes P450: cross-talk between the eicosanoid and endocannabinoid signaling pathways. Chem Rev. 2011;111:5899-921.

21. Green $\mathrm{K}$, Kearse EC, McIntyre OL. Interaction between delta9-tetrahydrocannabinol and indomethacin. Ophthalmic Res. 2001;33:217-20.

22. Green K, Podos SM. Antagonism of arachidonic acid-induced ocular effects by delta1-tetrahydrocannabinol. Invest Ophthalmol. 1974;13:422-9.

23. Hudson BD, Beazley M, Szczesniak AM, et al. Indirect sympatholytic actions at beta-adrenoceptors account for the ocular hypotensive actions of cannabinoid receptor agonists. J Pharmacol Exp Ther. 2011;339:757-67.

24. Plange N, Arend KO, Kaup M, et al. Dronabinol and retinal hemodynamics in humans. Am J Ophthalmol. 2007;143:173-4.

25. Nucci C, Bari M, Spano A, et al. Potential roles of (endo) cannabinoids in the treatment of glaucoma: from intraocular pressure control to neuroprotection. Prog Brain Res. 2008;173:451-64

26. Rapino C, Tortolani D, Scipioni L, Maccarrone, M. Neuroprotection by (endo)cannabinoids in glaucoma and retinal neurodegenerative diseases. Curr Neuropharmacol. 2018;16:959-70 
27. Goyal H, Awad HH, Ghali JK. Role of cannabis in cardiovascular disorders J Thorac Dis. 2017:9:2079-92.

AdelliGR, Bhagav P, Taskar P, et al Development of a deltagtetrahydrocannabinol amino acid-dicarboxylate prodrug with improved ocular bion:

29. Punyamurthula NS, Adelli GR, Gul W, et al. Ocular disposition of (8)-tetrahydrocannabinol from various topical ophthalmic formulations. AAPS PharmSciTech. 2017;18:1936-45.

30. Miller S, Kulkarni S, Ciesielski A, et al. Controlled-deactivation CB1 receptor ligands as a novel strategy to lower intraocular pressure Pharmaceuticals (Basel). 2018:11:50.

31. Szczesniak AM, Maor Y, Robertson $\mathrm{H}$, et al. Nonpsychotropic cannabinoids, abnormal cannabidiol and canabigerol- dimethyl heptyl, act at novel cannabinoid receptors to reduce intraocular pressure J OCul Pharmacol Ther. 2011:27:427-35. 32. Martinez-de-la-Casa JM, Garcia-Feijoo J, Fernandez-Vidal A, et al. Ocular response analyzer versus Goldmann applanation tonometry for intraocular pressure mea
Ophthalmol Vis Sci. 2006:47:4410-4. 